Communication

\title{
Single-Crystalline Fibers of Deuterated Potassium Dihydrogen Phosphate
}

\author{
Xueni Liu, Yan Ren *, Cheng Qian Zhang, Bo Wang and Sheng Qing Xia \\ State Key laboratory of Crystal Material, Shandong University, Jinan 250100, China; \\ 201812706@mail.sdu.edu.cn (X.L.); cqzhang@sdu.edu.cn (C.Q.Z.); wangbo1010@sdu.edu.cn (B.W.); \\ shqxia@sdu.edu.cn (S.Q.X.) \\ * Correspondence: ry@sdu.edu.cn
}

Received: 7 May 2020; Accepted: 13 June 2020; Published: 16 June 2020

\begin{abstract}
Single-crystalline fibers have distinct structures and optical properties comparing with the bulk crystals. In this article, two types of single-crystalline fibers of deuterated potassium

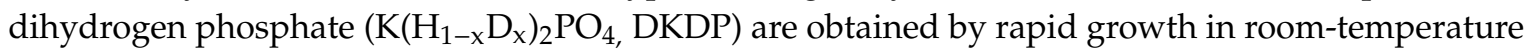
supersaturated solution. $\mathrm{X}$-ray diffraction analysis reveals that these DKDP single-crystalline fibers belong to tetragonal $(I-42 \mathrm{~d})$ and monoclinic $\left(P 2_{1} / \mathrm{c}\right)$ phases, respectively. The crystal structure of the tetragonal DKDP single-crystalline fiber is identical to that of the bulk DKDP tetragonal crystal reported. The lattice parameters of the monoclinic DKDP fiber (with the deuterium content of 55\%) are $\mathrm{a}=14.6571 \AA, \mathrm{b}=4.5187 \AA, \mathrm{c}=18.6962 \AA$, and $\beta=108.030^{\circ}$, which is a new crystal phase of DKDP. The monoclinic DKDP single-crystalline fiber is metastable at the present experimental condition and readily transit to the corresponding DKDP tetragonal phase in solution and in solid by grinding. The optical experiment shows that the highly deuterated tetragonal DKDP single-crystalline fiber possesses excellent optical guided-wave and effective second-harmonic generation properties. DKDP single-crystalline fibers are expected to be the suitable candidates for fabrication of the miniaturized nonlinear optical devices.
\end{abstract}

Keywords: single-crystalline fiber; deuterated potassium dihydrogen phosphate; crystal phase; phase transition; second harmonic generation

\section{Introduction}

Potassium dihydrogen phosphate $\left(\mathrm{KH}_{2} \mathrm{PO}_{4}, \mathrm{KDP}\right)$ and its isotope compound, deuterated potassium dihydrogen phosphate $\left(\mathrm{K}\left(\mathrm{H}_{1-\mathrm{x}} \mathrm{D}_{\mathrm{x}}\right)_{2} \mathrm{PO}_{4}, \mathrm{DKDP}\right)$, are irreplaceable electro-optical and nonlinear optical materials in the inertial confinement fusion (ICF) system because of their outstanding capability of growing into large size crystals with high optical quality as well as large nonlinear and electro-optic coefficients [1]. For the past few decades, therefore, the crystal growth and fabrication techniques are the main research fields for KDP-type crystals in order to satisfy the urgent demand of ICF for these two crystals [2].

Recently, we reported the crystal growth of one-dimensional KDP microstructures with a large length-to-diameter ratio grown under the ambient conditions. They exhibit unique crystal growth mechanism, which is quite different from that of the bulk KDP crystals. Moreover, a highly efficient second-harmonic generation was observed in these KDP single-crystalline fibers [3]. The discovery of these one-dimensional KDP materials opens a door to the unknown world of low-dimensional micro-/nano- KDP crystals. It indicates that except for the traditional bulk KDP/DKDP crystals, low-dimensions KDP-type crystals may become another group of robust nonlinear materials, especially for flexible and miniaturized device application in optical communications, high-energy laser, and so on. 
Crystal growth is the first problem before considering their device applications. The crystallization of a specific crystals is mainly controlled by the growth condition and molecular structure. For the isotopic crystals, on one hand, they generally have similar crystallization habits as well as phase species or phase structures under the same growth conditions. For example, DKDP crystallizes in the tetragonal crystal phase (I-42d) [4], which is isomophic to the KDP tetragonal phase formed at ambient temperature. On the other hand, the isotope deuterium plays a role in crystallization habit and formation of the crystal structure. The growth process of DKDP tetragonal phase usually accompanies the crystallization of the DKDP monoclinic phase $\left(P 2_{1}\right)[5,6]$. However, $P 2_{1}$ phase of DKDP has no isomophic KDP crystal phase crystallized in the aqueous solution. The structure of DKDP $P 2_{1}$ phase is similar to a monoclinic phase of KDP crystal formed at a relatively high temperature [7]. In this article, we study the crystallization habits of DKDP during the fast growth in high supersaturated aqueous solution at room temperature. Two types of single-crystalline fibers of DKDP with tetragonal (I-42D) and monoclinic $\left(P 2_{1} / \mathrm{c}\right)$ phases are obtained. The phase transition property between these two phases and the nonlinear frequency conversion property of the tetragonal DKDP single-crystalline fibers are also studied.

\section{Materials and Methods}

Hot solution was prepared using high purity KDP, $\mathrm{D}_{2} \mathrm{O}$ (99.9 atom\% deuterium, Aladdin, Shanghai, China) and ultrapure water (Millipore, America). All glasswares were washed before use in hydrochloric acid and then thoroughly washed with ultrapure water in an ultrasonic cleaner in order to remove the insoluble particles and possible metal ions (see Supplementary Materials) [8-10]. The hot DKDP solution is dropped on a cleaned glass slide, and evaporatively cooled at room temperature in a vacuum drier (Shuniu, Sichuan, China), which rapidly crystallizes and creates fiber-like DKDP single-crystals. The crystals are kept in vacuum dryer waiting for use. The final humidity of the vacuum drier is less than $10 \%$.

\section{Results and Discussion}

SEM (Scanning electron microscope) shows that the fibers obtained exhibit two types of shapes with polyhedral or tetragonal cross-section, respectively (see Figure 1). Moreover, the solid-core/tubular structures can be found in both types of fibers. The length-to-diameter ratio for both types of fiber is up to 500:1. The smooth prismatic surfaces of these fibers indicate an excellent crystal quality. Obviously, the shape of these single-crystalline fibers is very different to that of the bulk tetragonal or monoclinic phases of DKDP crystals previously reported [2,11].

The X-ray diffraction analysis is performed in order to determine the crystal structure of these single-crystalline fibers. The fiber crystals are picked from the sample freshly prepared, all of which are protected by the Paratone oil in order to keep away from the moisture during the measurement. The result shows that the DKDP single-crystalline fiber with the polygonal shape exhibits a $2 / \mathrm{m}$ point group symmetry with $P 2_{1} / \mathrm{c}$ space group, which is different to the structure of the bulk monoclinic DKDP crystal reported $\left(P 2_{1}\right)$. The summarized crystallorgraphic data of the monoclinic crystal phases of DKDP and KDP are shown in Table 1 for comparison. It shows that the unit cell parameters of the DKDP monoclinic single-crystalline fiber are $\mathrm{a}=14$.6571(7) $\AA, \mathrm{b}=4.5187(2) \AA, \mathrm{c}=18.6962(9) \AA$ and $\beta=108.030(2)^{\circ}$. The XRD spectra prove that the crystal structures of the polygonal shape DKDP fiber grown in different deuterium-content DKDP solution is the same monoclinic structure as shown in Table 1 (see Supplementary Materials). To our knowledge, the DKDP monoclinic $P 2_{1} / \mathrm{c}$ is a new crystal phase of DKDP. According to the data in Table 1 and the packing diagram shown in Figure 2, the structure of the DKDP $P 2_{1} / c$ phase is identical to that of the monoclinic KDP single-crystalline fiber $\left(P 2_{1} / \mathrm{c}\right)$ obtained under the same condition [3]. Therefore, the monoclinic phase DKDP single-crystalline fiber is isomorphic to the corresponding KDP monoclinic single-crystalline fiber crystal. The tetragonal-shaped single-crystalline fibers possess the identical space group and crystal lattice parameters to the bulk tetragonal DKDP phase (I-42d). Moreover, it is isomorphic to the 
KDP tetragonal phase [12]. Except for the above two crystal phases, no DKDP monoclinic phase $\left(P 2_{1}\right)$ was detected in the obtained DKDP crystals grown under the present experimental conditions [5].
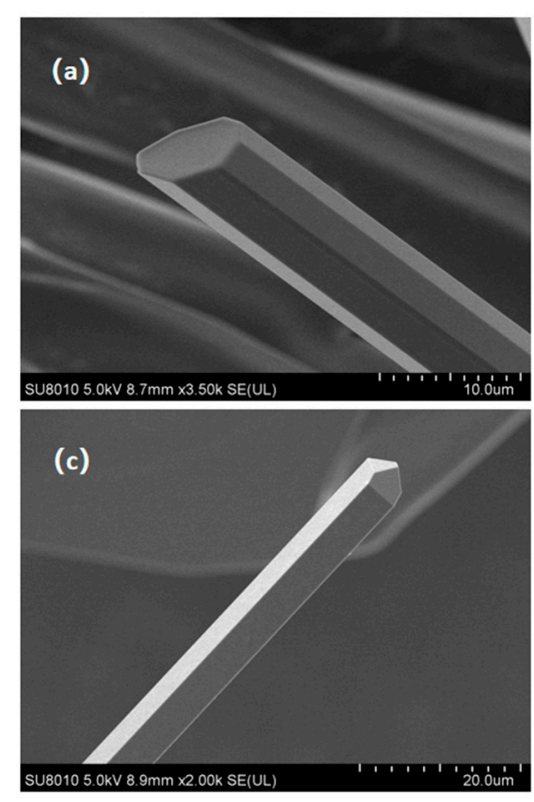
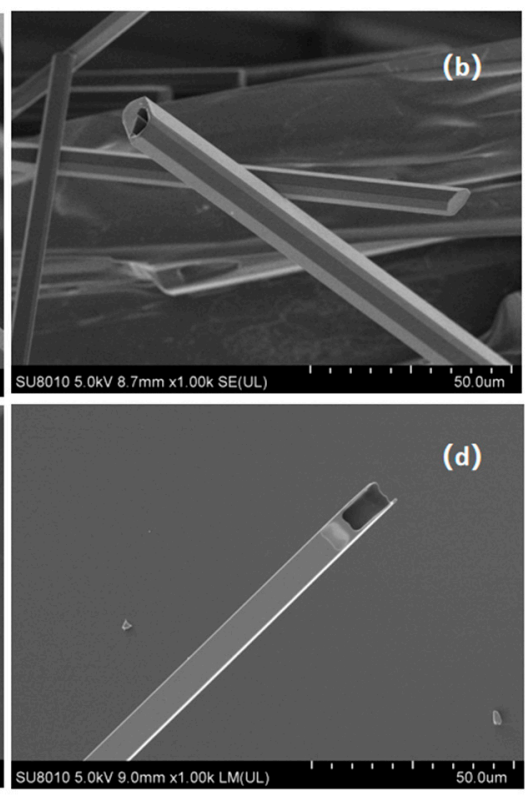

Figure 1. SEM images of the DKDP single-crystalline fibers obtained by rapid crystallization on glass substrate. $(\mathbf{a}, \mathbf{b})$ polyhedral solid-core/tubular single-crystalline fibers. $(\mathbf{c}, \mathbf{d})$ tetragonal solid-core/tubular single-crystalline fibers.

Table 1. Basic crystallographic data comparison of the monoclinic DKDP bulk crystal, monoclinic DKDP, and KDP single-crystalline fibers.

\begin{tabular}{cccc}
\hline Formula & $\mathbf{K}\left(\mathbf{H}_{\mathbf{0 . 1 6}} \mathbf{D}_{\mathbf{0 . 8 4}}\right)_{\mathbf{2}} \mathbf{P O}_{\mathbf{4}}$ & $\mathbf{K H}_{\mathbf{2}} \mathbf{P O}_{\mathbf{4}}$ & $\mathbf{K}\left(\mathbf{H}_{\mathbf{x}} \mathbf{D}_{\mathbf{1 - \mathbf { x }}} \mathbf{~}_{\mathbf{2}} \mathbf{P O}_{\mathbf{4}}\right.$ \\
\hline Space group & $P 2_{1}$ & $P 2_{1} / \mathrm{c}$ & $P 2_{1} / \mathrm{c}$ \\
$\mathrm{a}(\AA)$ & 7.1400 & $14.599(5)$ & $14.6571(7)$ \\
$\mathrm{b}(\AA)$ & 14.7100 & $4.503(5)$ & $4.5187(2)$ \\
$\mathrm{c}(\AA)$ & 7.4500 & $18.651(5)$ & $18.6962(9)$ \\
$\alpha\left(^{\circ}\right)$ & 90 & 90 & 90 \\
$\beta\left(^{\circ}\right)$ & 92.310 & $108.041(5)$ & $108.030(2)$ \\
$\gamma\left({ }^{\circ}\right)$ & 90 & 90 & 90 \\
$\mathrm{~V}\left(\AA^{3}\right)$ & 781.83 & $1165.8(14)$ & $1177.46(10)$ \\
$Z$ & 8 & 12 & 12 \\
$\mathrm{~T}(\mathrm{~K})$ & 293 & 293 & 297 \\
$\mathrm{CSD}$ No. & 34415 & 427178 & 1963699 \\
reference & {$[5]$} & {$[3]$} & This work \\
\hline
\end{tabular}

Raman spectra of the monoclinic and tetragonal single-crystalline fibers are measured in order to estimate their deuterium contents. The result is shown in Figure 3. The Raman spectrum of DKDP crystal typically possesses double peaks within $800 \mathrm{~cm}^{-1}$ to $1000 \mathrm{~cm}^{-1}$. The Raman peaks locate at $891.4 \mathrm{~cm}^{-1}$ and $964.5 \mathrm{~cm}^{-1}$ for the monoclinic phase single-crystalline fiber obtained in $75 \%$ deuterium content solution, while the peaks shift to $885.5 \mathrm{~cm}^{-1}$ and $987.6 \mathrm{~cm}^{-1}$ for the tetragonal single-crystalline fiber grown in solution containing $85 \%$ deuterium. The deuterium content for crystals is roughly estimated based on the peak maximum of $891.4 \mathrm{~cm}^{-1}$ and $888.5 \mathrm{~cm}^{-1}$ for monoclinc and tetragonal phases, respectively, based on the empirical formula [13]. The results are $55 \%$ for the monoclinic and $72 \%$ for the tetragonal single-crystalline fibers. In addition, the Raman scattering spectra are measured for multiple single-crystal fibers randomly chosen to show the deuterium-content fluctuation. The results are almost the same for these fiber samples. The statistical results for the deuterium-content estimation are shown Supplementary Materials. 


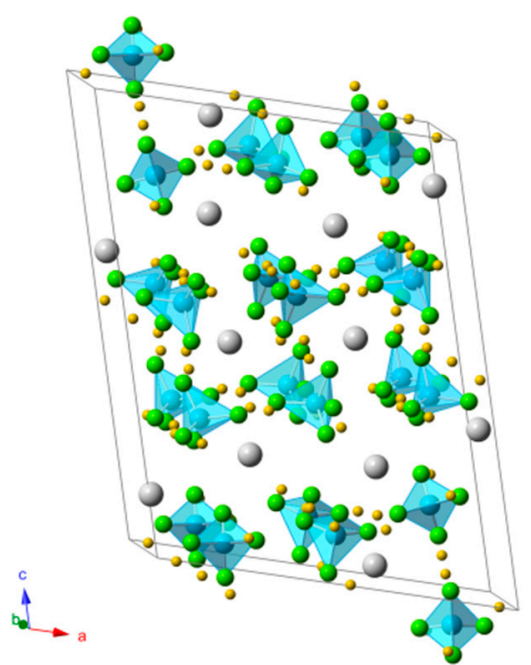

Figure 2. Packing diagram of monoclinic $\left(P 2_{1} / \mathrm{c}\right)$ DKDP crystal view along $\mathrm{b}$ axis. Grey: potassium, blue: phosphate, green: oxygen, yellow: hydrogen/deuterium.

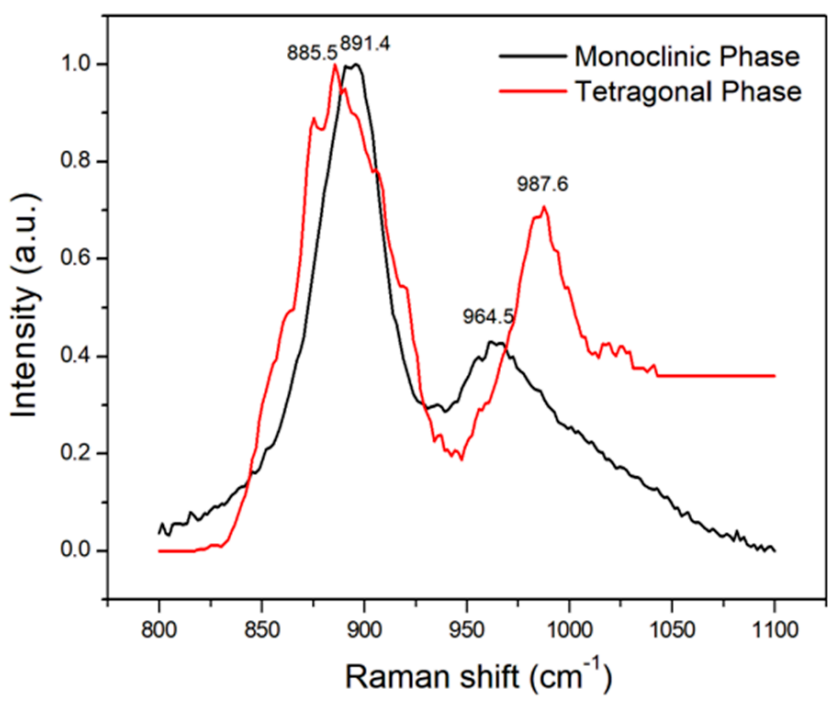

Figure 3. Raman spectra of the monoclinic phase single-crystalline fiber and tetragonal phase single-crystalline fiber. The double peaks indicate the existence of deuterium in the crystals.

Phase transition is one of the features of KDP-type crystals because of their hydrogen bond ferroelectric properties [14]. It is known that DKDP is characterized by the phase transition of the metastable tetragonal phase to the stable monoclinic phase $\left(P 2_{1}\right)$ [6]. However, according to the above description, there is no crystal phase of $P 2_{1}$ detected both in solution and in solid state crystals under the present condition. The main reason for an absence of bulk monoclinic phase being detected should be that the solution with the deuterium content of $75-85 \%$ is located at the stability region of the tetragonal phase. The phase equilibrium transition point for this deuterium content solution is higher than $60{ }^{\circ} \mathrm{C}$ [6]. Since our solution crystallizes typically at a temperature lower than $60{ }^{\circ} \mathrm{C}$, the bulk DKDP monoclinic phase is hardly detected in the solution. In our experiment, however, the monoclinic phase $\left(P 2_{1} / \mathrm{c}\right)$ DKDP fiber appears in the growth solution and the final products. We found that the monoclinic DKDP single-crystalline fiber readily transits to the tetragonal phase if there is tetragonal crystal nucleus or particles in the solution. Figure 4 shows the evolution process from the crystallization to dissolution of the monoclinic DKDP single-crystalline fibers. The monoclinic fibers begin to grow in Figure 4a. Then the tetragonal particles appear in Figure 4b, which grow rapidly as shown in Figure 4c. One can also see the gradual dissolution of the DKDP monoclinic single-crystalline fibers in 
Figure 4c. Finally, the tetragonal DKDP crystal particles grow much larger than before accompanied by the disappearance of the monoclinic DKDP single-crystalling fibers in Figure 4d. We further studied the XRD spectra of the crystallized fibers on the glass substrate. The result shows that the XRD spectra on the glass substrate belong to two crystal phases involving monoclinic and tetragonal phases (Figure 5a). However, the intensity of the featured peaks of the monoclinic single-crystalline fibers, such as $19.08^{\circ}$ and $26.9^{\circ}$, are decreased when the fibers on the substrates are grinded (Figure $5 b$ ). By contrast, the peak intensity of the tetragonal DKDP phase like $17.52^{\circ}, 23.96^{\circ}$, and $30.94^{\circ}$ is markedly increased (Figure $5 b$ ). Figure $5 c$,d show the XRD spectra of the pure DKDP tetragonal and monoclinic crystal phases, respectively. According to the above observation, therefore, the monoclinic DKDP single-crystalline fiber should be a metastable phase, and the tetragonal DKDP crystal phase is a stable phase under the present experimental condition.

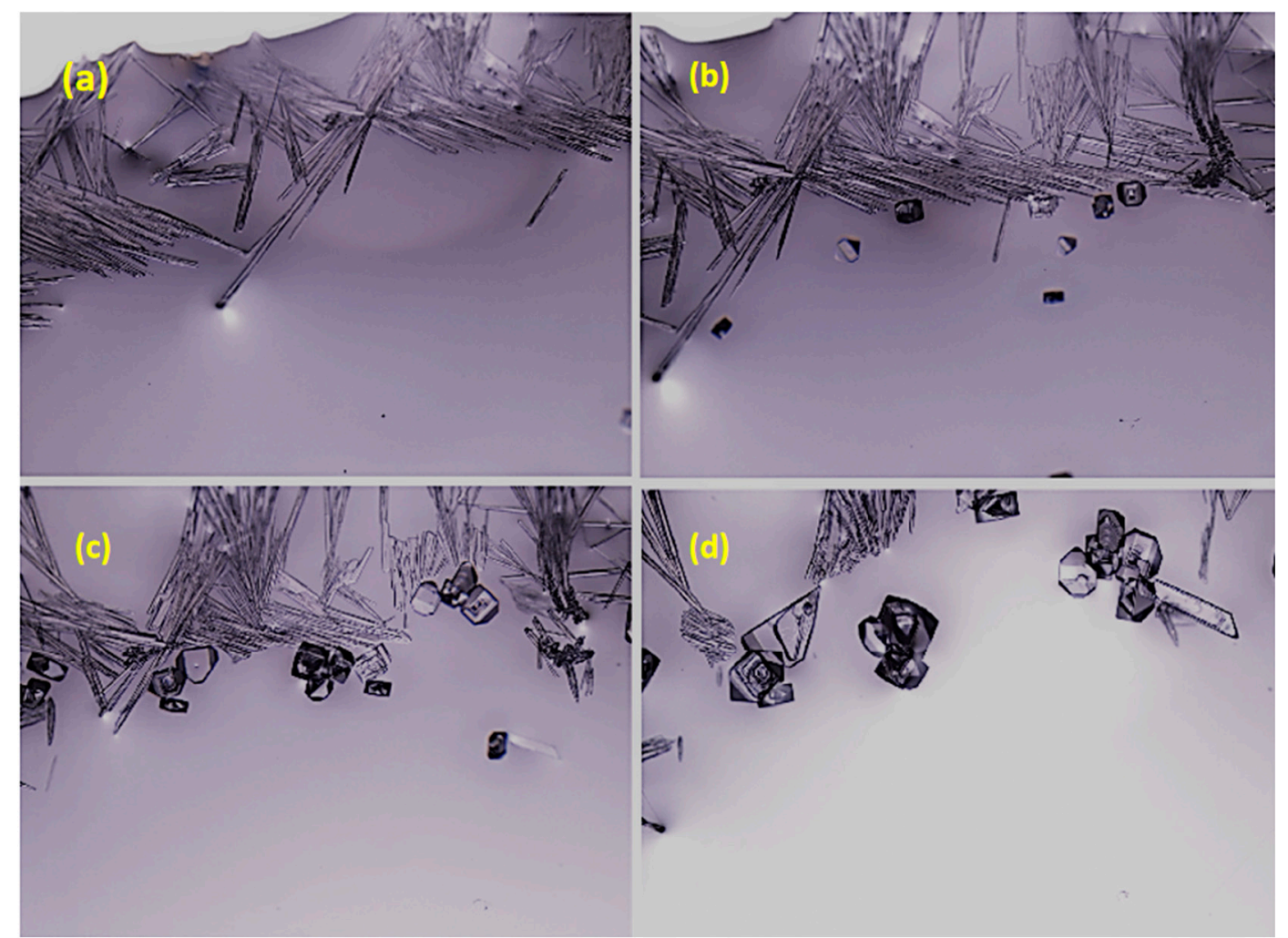

Figure 4. Phase transition of DKDP monoclinic single-crystal fibers as there is tetragonal phase present and growth in the solution. (a) the DKDP monoclinic single-crystal fibers growing in a solution droplet on glass substrate. (b) the small tetragonal crystal particles appear in the solution. (c) the tetragonal crystal particles grow rapidly accompanied by the dissolution of the monoclinic DKDP single-crystalline fibers. (d) the tetragonal crystals become large in size and the DKDP single-crystalline fibers disappear gradually.

The purpose of studying the DKDP low-dimensional crystals is to develop new functional materials with distinct optical properties. For single-crystalline fibers, they are characterized by the excellent nonlinearity, extended interaction length with laser light, tight spatial confinement of light in the waveguide structure, and natural physical flexibility, which benefit to high nonlinear conversion efficiency with much lower laser threshold [15-18]. Here, we further measure the second-harmonic generation (SHG) property of the tetragonal DKDP single-crystalline fiber since it is a non-centrosymmetric single-crystal (see the optical setup description in Supporting Information). Deuterated KDP crystals have the advantage over KDP in decreasing the damage possibility when they are used in type-II third-harmonic generation devices, which benefit from the split and reduced 
stimulated Raman scattering peaks as pumped by a high power laser [19]. In our experiment, the tetragonal single-crystalline fiber exhibits the excellent guided-wave for $532 \mathrm{~nm}$ laser (see Figure S4). No light leakage occurs during the laser propagating along the c-axial direction, which further indicates a high optical quality of the DKDP single-crystalline fiber. The second harmonic generation is achieved when a continuous wave $1064 \mathrm{~nm}$ laser being coupled into the entrance of the fiber along the c-axis. The spectrum is collected by a PMT grating spectrometer.

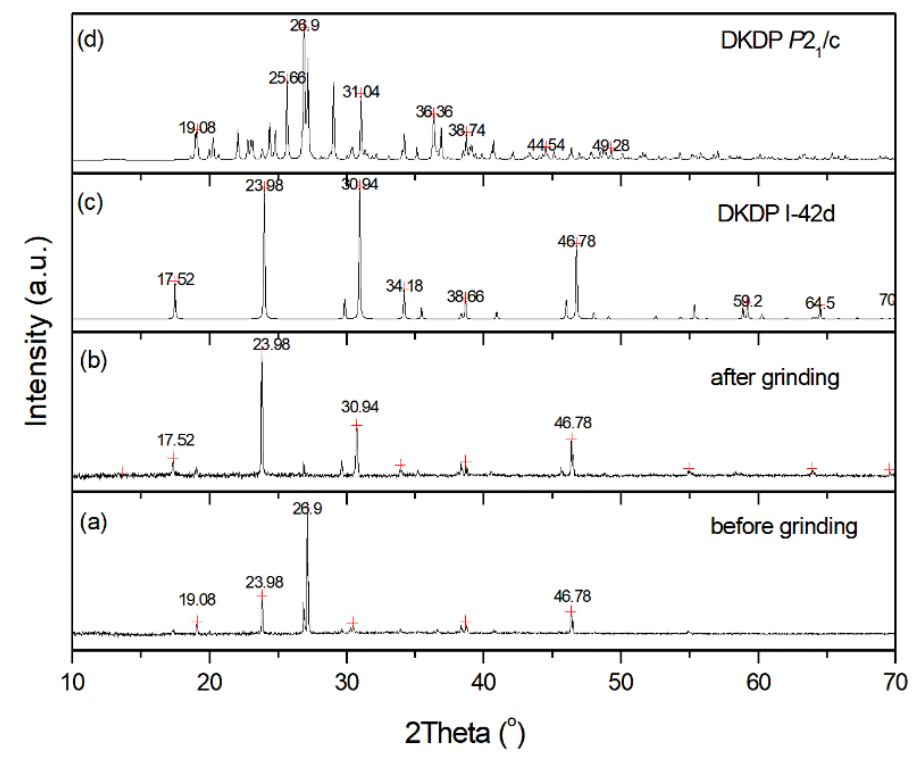

Figure 5. Comparison of XRD spectra of the DKDP monoclinic single-crystalline fibers before and after grinding. (a) the DKDP single-crystalline fibers on the substrate before grinding. (b) the DKDP single-crystalline fibers on the substrate after grinding. (c) the XRD spectra of tetragonal I-42d DKDP crystal phase. (d) the XRD spectrum of the monoclinic $P 2_{1} / c$ DKDP crystal phase.

The peak of the SHG spectrum in Figure 6a locates at $532.36 \mathrm{~nm}$ with the FWHM (full wide of half maximum) of $\sim 0.18 \mathrm{~nm}$. At the same time, a bright green light signal is captured by the CCD camera (Qimage MicroPublisherTM3.3 RTV, Olympus, Japan) opposite to the exit of the fiber (Figure 6a inset). The curve of the SHG output intensity versus input power $P(\omega)$ is shown in Figure $6 \mathbf{b}$, which is well fitted by the function of $P(2 \omega)=C_{2}{ }^{2} P^{2}(\omega)$. The fitting result shows a coherent propagation growth of the SHG along the c-axis of the fiber. The SHG efficiency calculated is about $5 \times 10^{-5} \mathrm{~W}^{-1}$. This frequency conversion efficiency is nearly the same level of that of the tetragonal KDP single-crystalline fiber we reported before [20]. The efficiency difference between them is not caused by the substitution deuterium for hydrogen in DKDP crystal. In fact, the diameter, length as well as the surface polishing play the key roles in the SHG efficiency improvement [21]. Here, the SHG of the tetragonal DKDP single-crystalline fiber should be an effective frequency conversion considering only about $2 \%$ of the pump light effectively has the right $k$ vector for efficient phase matching inside the fiber because of the spread of the $\mathrm{k}$. 

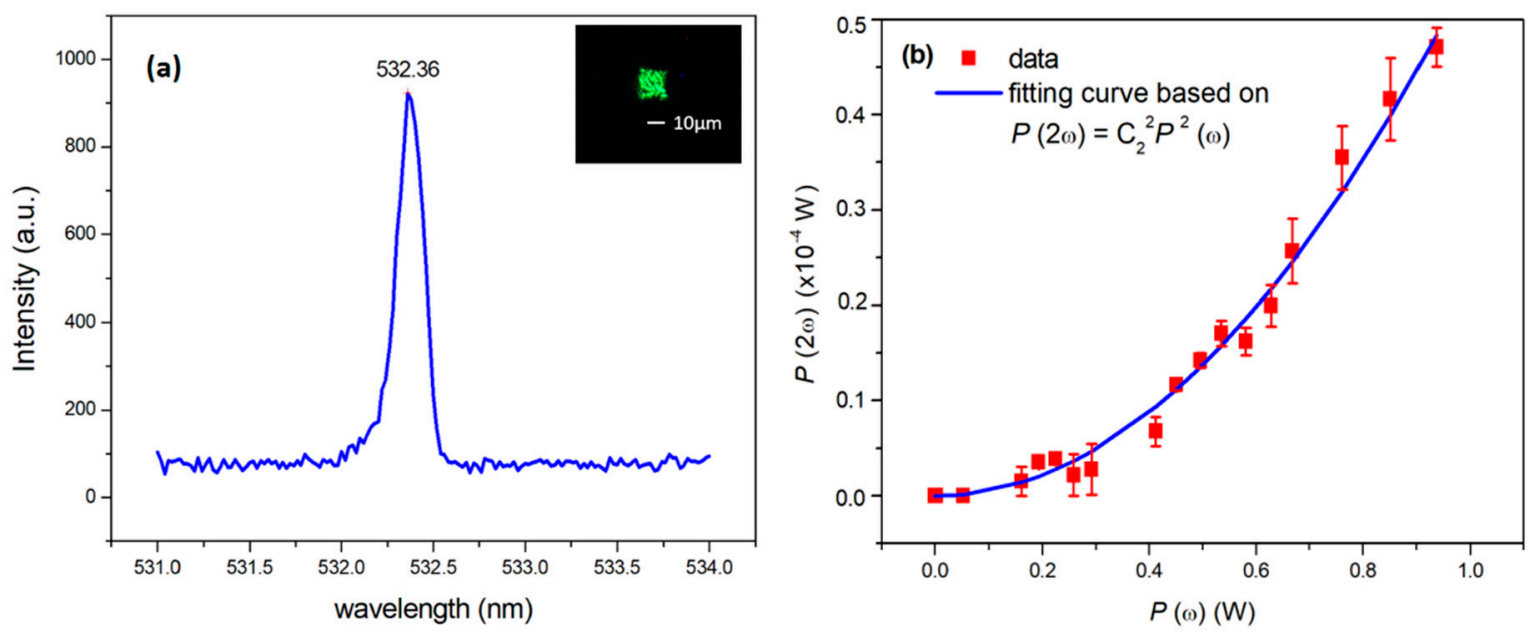

Figure 6. Second-harmonic generation (SHG) measurement of the tetragonal single-crystalline fiber. (a) the spectrum of the output SHG signal. The inset is the exit of the tetragonal fiber upon excitation by $1064 \mathrm{~nm}$ laser captured by the CCD camera. (b) SHG output intensity as a function of the pump power $P(\omega)$ from a solid-core crystal fiber with $\mathrm{D}=18 \mu \mathrm{m}$ and $\mathrm{L}=1.63 \mathrm{~mm}$. The red triangles are the experimental data, and the red solid line is the fitting curve based on $P(2 \omega)=C_{2}^{2} P^{2}(\omega)$.

\section{Conclusions}

In summary, DKDP single-crystalline fibers of the tetragonal and monoclinic phases have been obtained by the rapid growth of DKDP in solution under the ambient condition. The structure of the fibers has been solved. The monoclinic single-crystalline fiber is a new crystal phase of DKDP and isotomorphic to the monoclinic KDP single-crystalline fiber former reported [3]. The monoclinic DKDP single-crystalline fiber is also a meta-stable and readily transits to the corresponding tetragonal phase both in solution and in solid state by grinding. Our experiment showed that the tetragonal DKDP single-crystalline fiber is an effective frequency conversion crystal. Although without the phase-matching-direction crystal cutting [13], the coherent propagation growth of the SHG occurs along the c-axis. High effective frequency conversion output from the tetragonal DKDP single-crystalline fibers can be expected by further end-face polishing and coating.

Supplementary Materials: The following are available online at http://www.mdpi.com/2073-4352/10/6/511/s1, Figure S1: PXRD spectra of the monoclinic DKDP single-crystalline fibers grown in different deuterium-content solution, Figure S2: The deuterium content analysis of the DKDP monoclinic single-crystalline fibers based on Raman spectra, Figure S3: The deuterium content analysis of the DKDP tetragonal single-crystalline fibers, Figure S4: The optical guided-wave of the DKDP tetragonal single-crystalline fiber for $532 \mathrm{~nm}$ laser.

Author Contributions: Conceptualization, Y.R.; methodology, Y.R.; validation, Y.R., and X.L.; formal analysis, Y.R., X.L., C.Q.Z., B.W. and S.Q.X.; investigation, Y.R. and X.L.; writing-original draft preparation, Y.R. and X.L.; writing-review and editing, Y.R. and X.L.; visualization, Y.R.; supervision, Y.R.; project administration, Y.R.; funding acquisition, Y.R. All authors have read and agreed to the published version of the manuscript.

Funding: This work is sponsored by the financial supports of National Natural Science Foundation of China (Grant No. 51872163) and Natural Science Foundation of Shandong Province (ZR2019MEM006).

Acknowledgments: In this section you can acknowledge any support given which is not covered by the author contribution or funding sections. This may include administrative and technical support, or donations in kind (e.g., materials used for experiments).

Conflicts of Interest: There are no conflicts to declare.

\section{References}

1. de Yoreo, J.J.; Burnham, A.K.; Whitman, P.K. Developing $\mathrm{KH}_{2} \mathrm{PO}_{4}$ and $\mathrm{KD}_{2} \mathrm{PO}_{4}$ crystals for the world's most power laser. Int. Mater. Rev. 2002, 47, 113. [CrossRef] 
2. Zaitseva, N.; Garman, L. Rapid growth of KDP-type crystals. Prog. Cryst. Growth Charact. Mater. 2001, 43, 1. [CrossRef]

3. Ren, Y.; Zhao, X.; Deng, L.; Hagley, E.W. Ambient-condition of high-pressure phase centrosymmetric crystalline KDP microstructures for optical second harmonic generation. Sci. Adv. 2016, 2, e1600404. [CrossRef] [PubMed]

4. Kennedy, N.S.J.; Nelmes, R.J.; Thornley, F.R.; Rouse, K.D. Recent structural studies of the $\mathrm{KH}_{2} \mathrm{PO}_{4}-\mathrm{KD}_{2} \mathrm{PO}_{4}$ system. Ferroelectrics 1976, 14, 591-593. [CrossRef]

5. Nelmes, R.J. The crystal structure of monoclinic $\mathrm{KD}_{2} \mathrm{PO}_{4}$. Phys. Status Solidi B 1972, 52, K89. [CrossRef]

6. Jiang, M.H.; Fang, C.S.; Yu, X.L.; Wang, M.; Zheng, T.H.; Gao, Z.S. Polymorphism and metastable growth of DKDP. J. Cryst. Growth 1981, 53, 283. [CrossRef]

7. Itoh, K.; Matsurayashi, T.; Nakamura, E.; Motegi, H. X-ray study of high-temperature phase transitions in $\mathrm{KD}_{2} \mathrm{PO}_{4}$. J. Phys. Soc. Jpn. 1975, 39, 843. [CrossRef]

8. Rashkovich, L.N.; Kronsky, N.V. Influence of $\mathrm{Fe}^{3+}$ and $\mathrm{Al}^{3+}$ ions on the kinetics of steps on the $\{100\}$ faces of KDP. J. Cryst. Growth 1997, 182, 434. [CrossRef]

9. Wang, B.; Fang, C.; Wang, S.; Sun, X.; Gu, Q.; Li, Y.; Xu, X.; Zhang, J.; Liu, B.; Mou, X. The effects of Sn ${ }^{4+}$ ion on the growth habit and optical properties of KDP crystal. J. Cryst. Growth 2006, 297, 352. [CrossRef]

10. Mullin, J.W.; Amatavivadhana, A.; Chakraborty, M. Crystal habit modification studies with ammonium and potassium dihydrogen phosphate. J. Appl. Chem. 1970, 20, 153. [CrossRef]

11. Ravi, G.; Hameed, A.S.H.; Ramasamy, P. Effect of temperature and deuterium concentration on the growth of deuterated potassium dihydrogen phosphate (DKDP) single crystals. J. Cryst. Growth 1999, 207, 319. [CrossRef]

12. Dmitriev, V.G.; Gurzadyan, G.G.; Nikogosyan, D.N. Handbook of Nonlinear Optical Crystals; Springer-Verlag: Berlin, Germany, 1991.

13. Ji, S.; Teng, B.; Kong, W.; Zhong, D.; Sun, X.; Wang, Z. The effect of supersaturation levels on the segregation coefficient of deuterium in $\mathrm{K}\left(\mathrm{H}_{1-\mathrm{x}} \mathrm{D}_{\mathrm{x}}\right)_{2} \mathrm{PO}_{4}$ crystals using Raman spectroscopy. CrystEngComm 2016, $18,971$. [CrossRef]

14. Grünberg, J.; Levin, S.; Pelah, I.; Gerlich, D. High temperature phase transitions and metastability in KDP type crystals. Phys. Status Solidi B 1972, 49, 857. [CrossRef]

15. Jona, F.; Shirane, G. Ferroelectric Crystals; Dover: New York, NY, USA, 1993.

16. Yu, H.; Kim, D.Y.; Lee, K.J.; Oh, J.H. Fabrication of one-dimensional organic nanomaterials and their optoelectronic. J. Nanosci. Nanotechnol. 2014, 14, 1282. [CrossRef] [PubMed]

17. Zhou, H.; Wang, X.X.; Zhuang, X.J.; Pan, A.L. Second harmonic generation and waveguide properties in perovskite $\mathrm{Na}_{0.5} \mathrm{~B}_{\mathrm{i} 0.5} \mathrm{Ti}_{3}$ Nanowires. Opt. Lett. 2016, 41, 3803. [CrossRef] [PubMed]

18. Schiek, R.; Pertsch, T. Absolute measurement of the quadratic nonlinear susceptibility of lithium niobate in waveguides. Opt. Mater. Express 2012, 2, 126. [CrossRef]

19. Hou, Y.C.; Pan, S.K. Nonlinear optical crystals and their applications. J. Synth. Cryst. 1990, 19, 356.

20. Ren, Y.; Zhang, S.J. Optical quality tetragonal phase single-crystal fiber of potassium di-hydrogen phosphate with efficient second-harmonic generation. CrystEngComm 2017, 19, 767. [CrossRef]

21. Yang, H.W.; Song, J.W.; Li, D.Z.; Li, X.F.; Cheng, W.Y.; Ren, Y. Nanosecond-pusled frequency-doubled laser in monoclinic single-crystalline KDP microstrutures. IEEE Photon. Technol. Lett. 2019, 31, 1080. [CrossRef]

(C) 2020 by the authors. Licensee MDPI, Basel, Switzerland. This article is an open access article distributed under the terms and conditions of the Creative Commons Attribution (CC BY) license (http://creativecommons.org/licenses/by/4.0/). 